\title{
Multicore Fiber Sensors for Simultaneous Measurement of Force and Temperature
}

\author{
Amy Van Newkirk, J. Enrique Antonio-Lopez, Guillermo Salceda-Delgado, \\ Mohammad Umar Piracha, Rodrigo Amezcua-Correa, and Axel Schülzgen
}

\begin{abstract}
We demonstrate a force and temperature sensor consisting of a multicore fiber (MCF) spliced between two single-mode fibers. Increasing of the sensitivity to applied force is achieved through etching of the MCF cladding, with an overall increase of $7 x$ compared with a $125-\mu \mathrm{m}$ MCF device, and 12x compared with a standard fiber Bragg grating (FBG). Simultaneous decoupling of force and temperature with high accuracy is demonstrated using two MCF sections with different outer diameters. This device has robust operation up to $1000{ }^{\circ} \mathrm{C}$, making it more suitable than FBGs for extreme environments.
\end{abstract}

Index Terms-Fiber optics, optical fiber devices, optical fiber sensors.

\section{INTRODUCTION}

$\mathbf{F}$ IBER optics are widely used for sensing in a broad range of applications, including temperature, refractive index, liquid level, pressure, acoustic waves, strain, etc. Particularly for the cases of strain and temperature measurements, fiber Bragg gratings (FBGs) have dominated for being relatively simple, reliable, and well-suited for many applications [1]. Additionally, FBGs have been used in configurations for decoupling simultaneous strain and temperature measurements [2], [3]. However, traditional FBGs are limited by the environments in which they can operate, as most degrade at temperatures above a few hundred degrees Celsius. Alternative fiber optic sensors operating on the principle of multimode interference (MMI) have been also shown to stably measure both strain and temperature [4], [5]. Many MMI fiber devices consist of a multimode fiber spliced between two single mode fibers (SMFs), sometimes known as SMS devices [6]-[8].

Here we show the application of a sensor based on multicore fiber (MCF) for high sensitivity force and strain sensing.

Manuscript received March 4, 2015; revised April 15, 2015; accepted April 21, 2015. Date of publication April 29, 2015; date of current version June 22, 2015. This work was supported in part by the Florida High Tech Corridor Council and in part by FAZ Technology, Inc., Orlando, FL, USA. The work of G. Salceda-Delgado was supported by the National Council of Science and Technology (CONACYT).

A. Van Newkirk, J. E. Antonio-Lopez, R. Amezcua-Correa, and A. Schülzgen are with the College of Optics and Photonics, University of Central Florida, Orlando, FL 32816 USA (e-mail: amy.vannewkirk@ knights.ucf.edu; jealopez@creol.ucf.edu; r.amezcua@creol.ucf.edu; axel@creol.ucf.edu).

G. Salceda-Delgado is with the College of Optics and Photonics, University of Central Florida, Orlando, FL 32816 USA, and also with Centro De Investigaciones en Optica, A.C., León 37150, Mexico (e-mail: guillermo.salcedadelgado@ucf.edu).

M. U. Piracha was with FAZ Technology, Inc., Orlando, FL 32826 USA (e-mail: upiracha@hotmail.com).

Color versions of one or more of the figures in this letter are available online at http://ieeexplore.iee.org.

Digital Object Identifier 10.1109/LPT.2015.2427733

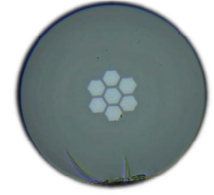

(a)

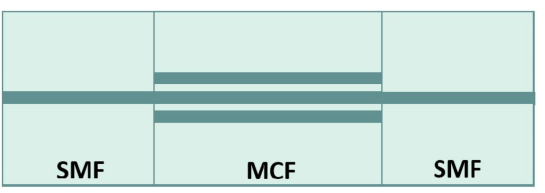

(b)
Fig. 1. a) Microscope image of the 7 core fiber used. b) Diagram of the MCF device structure.

Recently, Zhong et al. showed an increase of the strain sensitivity of long period fiber Bragg gratings through inflating the gratings with a $\mathrm{CO}_{2}$ laser. While effective, this approach is complex and achieved a maximum sensitivity of $-5.62 \mathrm{~nm} / \mathrm{m} \varepsilon$ [9]. In a much simpler and more reproducible approach, we look at the dependence of the sensitivity on the cladding diameter of the MCF. A dependence of strain sensitivity on the outer diameter (OD) of an FBG has previously been studied by Mondal et al. [10]. Additionally, we show decoupling of temperature and force measurements using two devices with different outer diameters (ODs).

Recently, a fiber optic sensor consisting of a section of MCF spliced between two pieces of SMF was shown to stably operate at temperatures up to $1000{ }^{\circ} \mathrm{C}$ [11]. A seven core fiber can support seven polarization degenerate supermodes, but when excited by the fundamental mode of SMF, only 2 supermodes are excited. These modes interfere within the MCF section, producing a periodically modulated transmission spectrum. The modal properties of this seven-core fiber have been fully explored and optimized for maximum transmission modulation [12]. The MCF device transmission spectrum was previously shown to shift with applied temperature due to a change in refractive index, according to the thermo-optic coefficient. Here we show that this device is also sensitive to strain, as the interference pattern shifts with both the refractive index change and the fiber elongation caused by an applied force.

\section{FORCE MEASUREMENT}

The MCF used was fabricated in-house, and a cross-section is shown in Fig. 1 (a). It has 7 germanium-doped coupled cores with diameters of $10.6 \mu \mathrm{m}$, a pitch of $12.1 \mu \mathrm{m}$, an NA of 0.13 , and an outer diameter of $125 \mu \mathrm{m}$. Shown in Fig. 1 (b) is a diagram of the device utilizing MCF. The length of MCF used in all following experiments is $2 \mathrm{~cm}$, and SMF-28e is used as the SMF. The insertion loss of the device has been measured to be less than $0.05 \mathrm{~dB}$. 


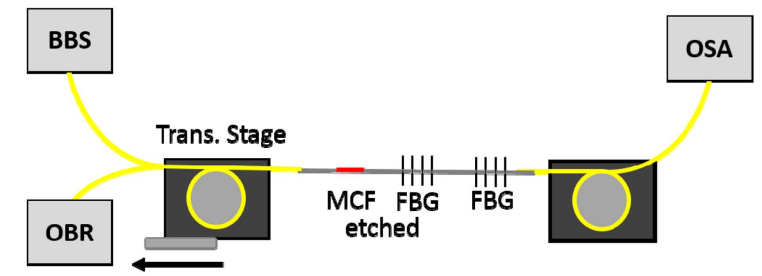

Fig. 2. Schematic of experimental setup.

To measure the force and strain sensitivity of the MCF device, a fiber chain was created and placed in the setup shown in Fig. 2. The fiber chain consisted of several elements in order to accurately compare the sensitivity of the MCF sensor with FBG based devices. The chain included a standard FBG with $125 \mu \mathrm{m}$ OD to be used as a reference, a second FBG with an etched cladding, and a section of MCF with an etched cladding. Fiber etching was done in 6:1 buffered oxide etch (BOE), at a rate of $0.24 \mu \mathrm{m} /$ minute. The FBG and MCF segment were etched together in order to ensure the same final OD. The FBGs used were Draw Tower Gratings (FBGS Technologies) with a reflectivity of $20 \%$ near $1555 \mathrm{~nm}$. The fiber chain was then spliced to $900 \mu \mathrm{m}$ jacketed patchcords, which were then wound around $4 \mathrm{~cm}$ diameter posts to ensure no slipping of the fiber as it was stretched. One post was fixed, while the other was attached to a translation stage, allowing for longitudinal force to be applied to the fiber through movement of the stage. A supercontinuum source (NKT SuperK Compact) was used as the broad band light source (BBS), and the transmission spectrum was monitored with an optical spectrum analyzer (OSA). The FBG's reflections were simultaneously measured using an optical backscatter reflectometer (OBR).

As force is applied to the MCF segment, the fiber elongates and the refractive index changes. This causes a shift in the transmission spectrum of the MCF device as shown in Fig. 3(a), similar to that previously measured as a function of temperature [11]. Due to the applied force the reflection maxima of the FBGs also shifted as shown in Fig. 3(b). The reference FBG's wavelength shift was used to calculate the applied force using the well-known relations given in [1].

Because all three sensors are in a single chain, they experience the same force when the translation stage is moved. However, due to the different ODs, the stress on each section is different, because stress is equal to force/area. A smaller crosssectional area of the fiber produces a higher stress, which leads to a higher strain, according to Young's modulus as shown here:

$$
E=\frac{\sigma}{\varepsilon}=\frac{F / A}{\Delta l / l}
$$

where $E$ is Young's Modulus, which is $73 \mathrm{GPa}$ for fused silica, $\sigma$ is stress, $\varepsilon$ is strain, $F$ is force, $A$ is cross-sectional area, $l$ is the fiber length, and $\Delta l$ its change due to the applied force. Therefore a fiber with a smaller diameter experiences a higher strain for a given applied force. This higher strain leads to a larger wavelength shift in transmission. This was observed for the etched fiber devices. An example plot of wavelength shift vs. force for all sensors within the chain is shown in Fig. 4. Due to the fact that force is the constant factor

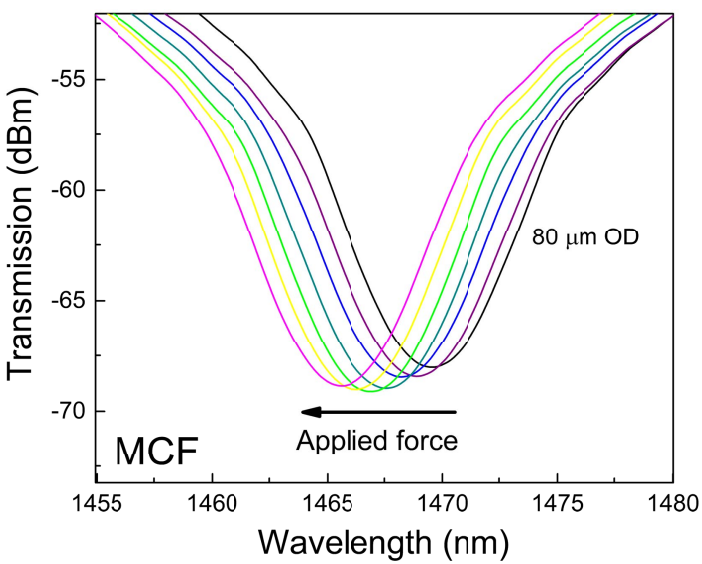

(a)

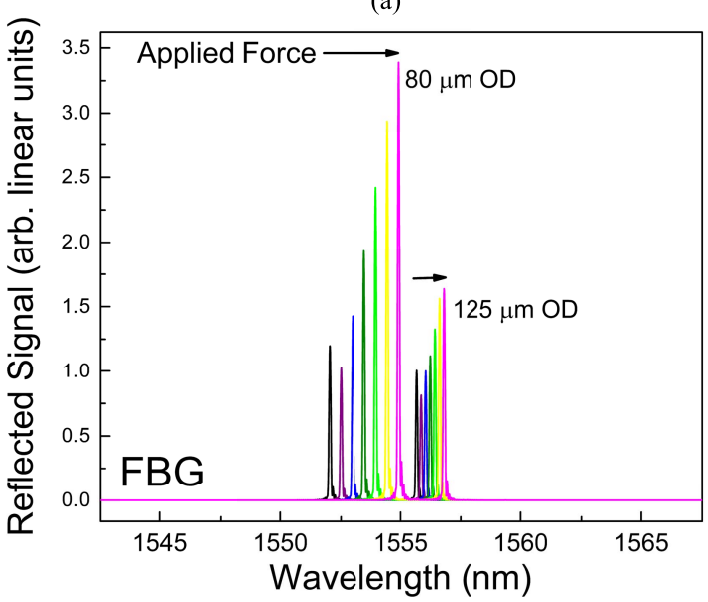

(b)

Fig. 3. Force-induced spectral shift of MMI minima of a MCF device with $80 \mu \mathrm{m}$ OD (a) and two FBGs with $80 \mu \mathrm{m}$ and $125 \mu \mathrm{m}$ ODs (b) from 0 to 0.65 Newtons of applied force. The MCF was measured in transmission, while the FBGs were measured in reflection with an OBR.

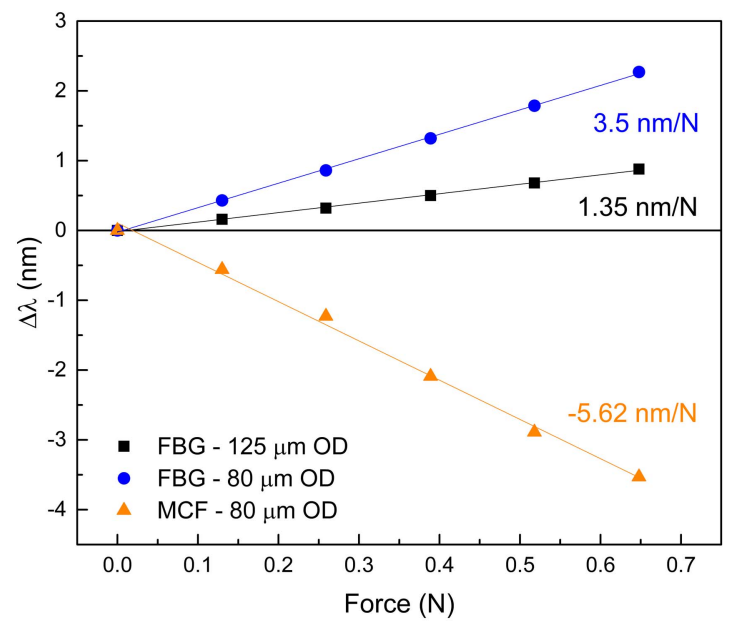

Fig. 4. Spectral shift vs. applied force for 3 sensors in chain.

in the fiber chains, we will refer to force sensitivities, not strain sensitivities of the devices; however the strain sensitivity can be easily calculated for each diameter using Eqn. 1. The strain sensitivities of the devices are constant with respect to OD and are $1.2 \mathrm{~nm} / \mathrm{m} \varepsilon$ and $-2.1 \mathrm{~nm} / \mathrm{m} \varepsilon$ for the FBG and MCF devices, respectively.

While the strain sensitivity is constant, the force sensitivity is a function of the fiber sensor OD, and the measured depen- 


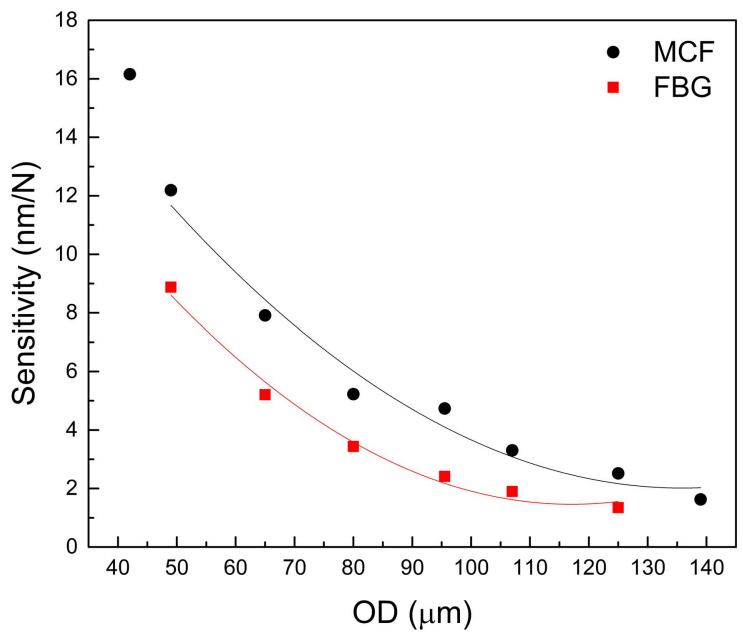

Fig. 5. Force sensitivity as a function of fiber OD with a parabolic fit for the MCF and FBG sensors.

dency of several MCF and FBG devices is shown in Fig. 5. A parabolic fit was then applied to the data, showing that the force sensitivity is proportional to $\left(1 / \mathrm{OD}^{2}\right)$. This agrees with the dependence of applied stress on the cross-sectional area of the fiber for a given force. Only the measured sensitivity of the MCF device with the smallest OD, $42 \mu \mathrm{m}$, appears higher than the parabolic fit. This is assumed to be due to a change in the supermodes of the MCF. At an OD of $42 \mu \mathrm{m}$ the outer core to cladding distance is only 3.6 microns. This causes the supermodes to overlap with the outside of the fiber, influencing the effective indices and mode profiles of the supermodes for the smallest investigated OD. Decreasing the OD of the MCF is a simple way to increase the sensitivity of the sensor to force, and can be done through fiber etching or initially fabricating a MCF with a smaller cladding. The $42 \mu \mathrm{m}$ MCF sensor had an overall sensitivity increase of $7 x$ that of a $125 \mu \mathrm{m}$ MCF device, and 12x that of a standard FBG. Etched FBGs showed a similar increase in sensitivity, also following a $\left(1 / \mathrm{OD}^{2}\right)$ dependence, but were consistently about $40 \%$ less sensitive than the MCF devices with the same OD.

\section{COMBINED EXPERIMENT}

Because the sensitivity of the MCF device to longitudinal force can be easily tuned through the OD, two devices can be used in combination to decouple force and temperature measurements. This was demonstrated using a similar setup as shown in Fig. 2, but with the fiber chain now consisting of a standard $125 \mu \mathrm{m}$ OD FBG for reference again, a $125 \mu \mathrm{m}$ OD section of MCF, and an $80 \mu \mathrm{m}$ OD section of MCF. Also, the two MCF segments were suspended in glass capillaries over hot plates for temperature tuning.

The transmission spectra of the two MCF devices is shown in Fig. 6(a). Splicing the two devices together into a chain creates the transmission spectrum shown in Fig. 6(b). Here, both MMI minima are still clearly visible, however this chain could be further optimized by slightly tuning the length of the MCF sections to further separate the MMI minima from each other. The dependence of the transmission minima on the MCF length was calculated by Jollivet et al. [13]. Both MCF devices

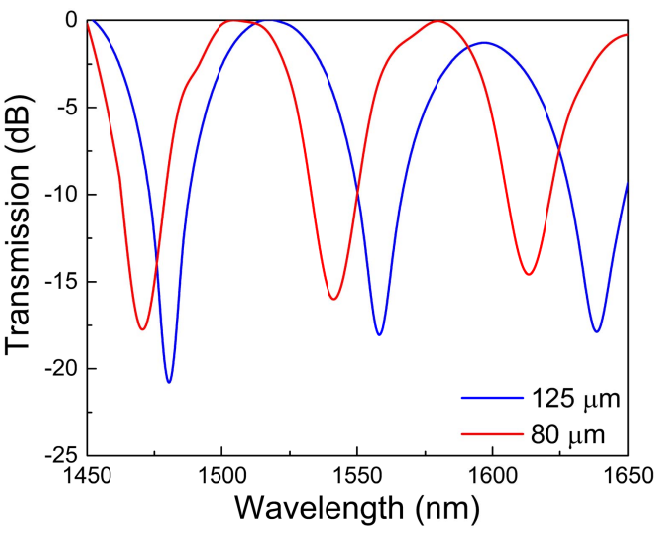

(a)

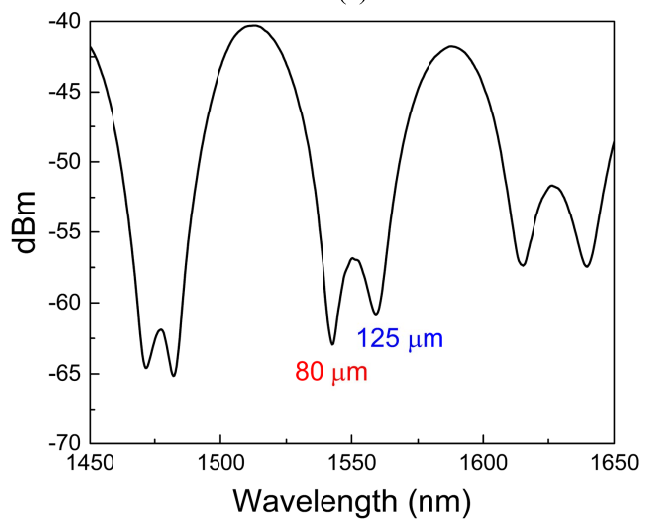

(b)

Fig. 6. (a) Transmission spectra of individual MCF devices and (b) both devices in a chain.

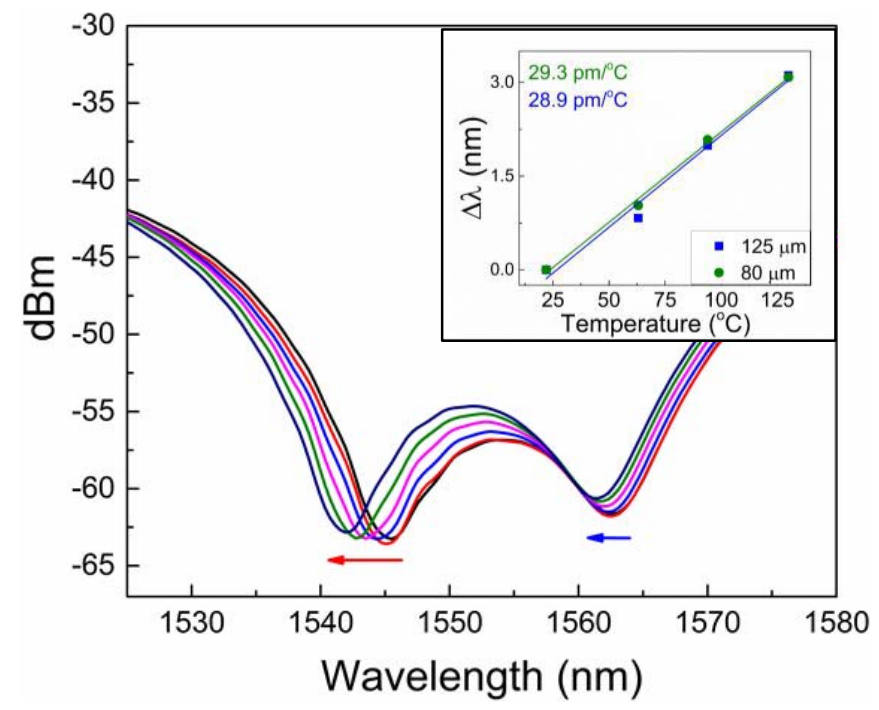

Fig. 7. Spectral shift of $80 \mu \mathrm{m}$ (red) and $125 \mu \mathrm{m}$ (blue) OD MCF devices with applied force. (inset) Measured temperature sensitivity of MCF devices.

can then be individually monitored using a single transmission measurement. Shown in Fig. 7 are the spectra of the device chain as force is applied. Clearly, the $80 \mu \mathrm{m}$ device is shifting more than the $125 \mu \mathrm{m}$ device, due to the increased force sensitivity shown previously.

The temperature sensitivity of each device was also measured using hot plates and was found to be $29 \mathrm{pm} /{ }^{\circ} \mathrm{C}$, equal 


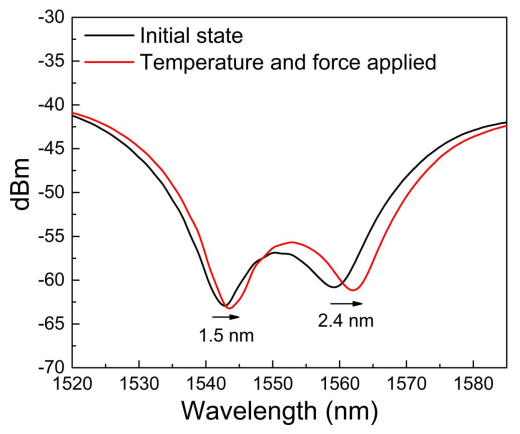

Fig. 8. Spectral shift with both force and temperature applied.

for both devices within the measurement error of about $2 \%$, as shown in the inset of Fig. 7. Here, the OD has no influence on the sensitivity of the MCF to temperature, because the temperature shift is caused by the refractive index change in the fiber according to the thermo-optic coefficient, and not the photo-elastic effect or the negligible fiber elongation.

Next, a combination of longitudinal force and temperature were applied to the fiber chain containing both MCF segments and the reference FBG. Both transmission minima shifted in wavelength, but by different amounts, due to the variation in strain. This is shown in Fig. 8. To determine both the strain and the temperature experienced by the MCF devices, the following equations can be used:

$$
\begin{aligned}
\Delta \lambda_{80} & =a \Delta F+c \Delta T \\
\Delta \lambda_{125} & =b \Delta F+c \Delta T
\end{aligned}
$$

where $a$ and $b$ are the force sensitivities shown in Fig. 4, and $c$ is the temperature sensitivity listed above. The measured wavelength shifts were $1.5 \mathrm{~nm}$ and $2.4 \mathrm{~nm}$ for the $80 \mu \mathrm{m}$ and $125 \mu \mathrm{m}$ OD devices, respectively. Using the above equations, the calculated temperature shift $(\Delta \mathrm{T})$ was $112^{\circ} \mathrm{C}$. With an initial starting temperature of $22^{\circ} \mathrm{C}$, adding this to $\Delta \mathrm{T}$ gives a calculated temperature of $134^{\circ} \mathrm{C}$. From a thermal image (using FLIR E60) of the hotplates, the applied temperature was measured to be $133^{\circ} \mathrm{C}$. This is within $1 \%$ of the experimental measurement. The measured force was $0.33 \mathrm{~N}$; this force corresponds to a strain of $0.905 \mathrm{~m} \varepsilon$ for an OD of $80 \mathrm{um}$, and $0.371 \mathrm{~m} \varepsilon$ for $125 \mu \mathrm{m} \mathrm{OD}$. The FBG that was also in the fiber chain measured a strain of $0.381 \mathrm{~m} \varepsilon$, within $3 \%$ of the value calculated for a $125 \mu \mathrm{m}$ OD.

The MCF sensors were then strength tested for high stress environment measurements. Using $125 \mu \mathrm{m}$ OD fiber, MCF to SMF splices were stressed in a similar setup to Fig. 2, until the splice breaking point. This test was repeated several times and compared to SMF to SMF splices in the same setup. It was found that the MCF-SMF splices survived to an average strain of $7.11 \mathrm{~m} \varepsilon$, and SMF-SMF splices survived to $7.55 \mathrm{~m} \varepsilon$. This shows that the MCF-SMF splices are just as strong as typical SMF-SMF splices, and they are robust enough to withstand environments with up to several millistrain applied. It is important to note that decreasing the outer diameter may in turn decrease the strength of the fiber, as the $42 \mu \mathrm{m}$ OD sensor only withstood up to $3 \mathrm{~m} \varepsilon$ before failure, but this has not been fully investigated.

\section{CONCLUSION}

In conclusion, we have demonstrated the use of a MCF spliced between 2 SMFs as a force and strain sensor. This device has previously been shown to withstand temperatures up to $1000^{\circ} \mathrm{C}$, making it potentially more suitable than FBGs for elevated temperature measurements for applications such as down-hole oil drilling or nuclear reactor facilities. Also, we have shown the enhancement of the longitudinal force sensitivity through simple etching of the cladding of the device. Using this variation in sensitivity, simultaneous decoupling of force and temperature measurements was demonstrated, allowing both quantities to be determined with high accuracy using a single transmission spectrum. MCF-based sensors are simple and inexpensive to fabricate, requiring only two standard splices between SMF and MCF, making these devices an attractive and robust alternative to traditional fiber sensors for applications of strain and temperature measurement. Additionally, the symmetry of the seven core fiber design greatly reduces the polarization dependence that must be taken into consideration when using most other devices based on multicore or few-mode fibers.

\section{REFERENCES}

[1] A. Kersey et al., "Fiber grating sensors," J. Lightw. Technol., vol. 15, no. 8, pp. 1442-1463, Aug. 1997.

[2] B.-O. Guan, H.-Y. Tam, X.-M. Tao, and X.-Y. Dong, "Simultaneous strain and temperature measurement using a superstructure fiber Bragg grating," IEEE Photon. Technol. Lett., vol. 12, no. 6, pp. 675-677, Jun. 2000.

[3] J. Jung, H. Nam, J. H. Lee, N. Park, and B. Lee, "Simultaneous measurement of strain and temperature by use of a single-fiber Bragg grating and an erbium-doped fiber amplifier," Appl. Opt., vol. 38, no. 13, pp. 2749-2751, May 1999.

[4] Y. Liu and L. Wei, "Low-cost high-sensitivity strain and temperature sensing using graded-index multimode fibers," Appl. Opt., vol. 46, no. 13, pp. 2516-2519, May 2007.

[5] S. M. Tripathi, A. Kumar, R. K. Varshney, Y. B. P. Kumar, E. Marin, and J.-P. Meunier, "Strain and temperature sensing characteristics of single-mode-multimode-single-mode structures," J. Lightw. Technol., vol. 27, no. 13, pp. 2348-2356, Jul. 1, 2009.

[6] A. Kumar, R. K. Varshney, S. Antony C., and P. Sharma, "Transmission characteristics of SMS fiber optic sensor structures," Opt. Commun., vol. 219, nos. 1-6, pp. 215-219, 2003.

[7] Q. Wu, Y. Semenova, A. M. Hatta, P. Wang, and G. Farrell, "Single-mode-multimode-single-mode fiber structures for simultaneous measurement of strain and temperature," Microw. Opt. Technol. Lett., vol. 53, no. 9, pp. 2181-2185, Sep. 2011.

[8] J. R. Guzman-Sepulveda and D. A. May-Arrioja, "In-fiber directional coupler for high-sensitivity curvature measurement," Opt. Exp., vol. 21, no. 10, pp. 11853-11861, May 2013.

[9] X. Zhong et al., "High-sensitivity strain sensor based on inflated long period fiber grating," Opt. Lett., vol. 39, no. 18, pp. 5463-5466, Sep. 2014.

[10] S. K. Mondal et al., "Single fiber Bragg grating sensor with two sections of different diameters for longitudinal strain and temperature discrimination with enhanced strain sensitivity," Rev. Sci. Instrum., vol. 80, no. 10, p. 103106, Oct. 2009

[11] J. E. Antonio-Lopez, Z. S. Eznaveh, P. LiKamWa, A. Schülzgen, and R. Amezcua-Correa, "Multicore fiber sensor for high-temperature applications up to $1000^{\circ} \mathrm{C}$, 'Opt. Lett., vol. 39, no. 15, pp. 4309-4312, Aug. 2014.

[12] A. Van Newkirk, E. Antonio-Lopez, G. Salceda-Delgado, R. Amezcua-Correa, and A. Schülzgen, "Optimization of multicore fiber for high-temperature sensing," Opt. Lett., vol. 39, no. 16, pp. 4812-4815, Aug. 2014.

[13] C. Jollivet et al., "Mode-resolved gain analysis and lasing in multisupermode multi-core fiber laser," Opt. Exp., vol. 22, no. 24, pp. 30377-30386, Nov. 2014. 